
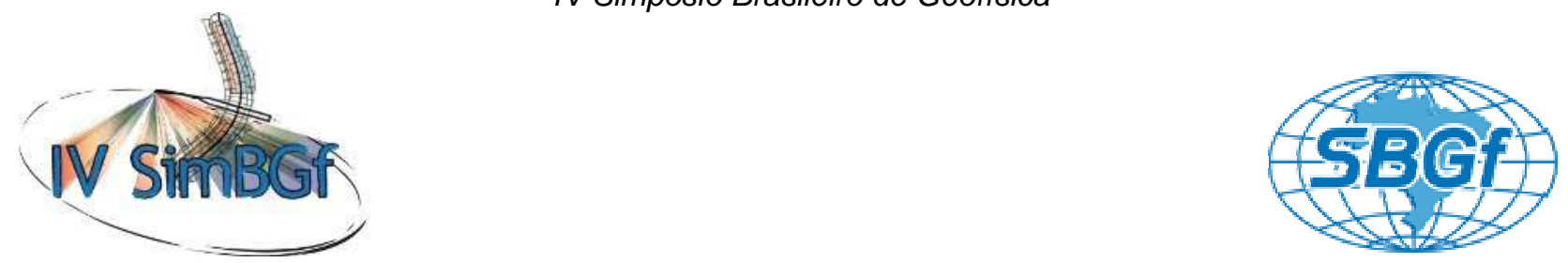

\title{
Possíveis correlações de padrões harmônicos entre séries históricas de dados geomagnéticos e de atividade solar no Brasil
}

\author{
Daniel R. Franco*, Fillipe C. L. Siqueira, Sérgio L. Fontes, Observatório Nacional (ON-MCT)
}

Copyright 2010, SBGf - Sociedade Brasileira de Geofísica

Este texto foi preparado para a apresentação no IV Simpósio Brasileiro de Geofísica, Brasília, 14 a 17 de novembro de 2010. Seu conteúdo foi revisado pelo Comitê Técnico do IV SimBGf, mas não necessariamente representa a opinião da SBGf ou de seus associados. É proibida a reprodução total ou parcial deste material para propósitos comerciais sem prévia autorização da SBG.

\begin{abstract}
Neste trabalho, discutimos resultados preliminares de um estudo baseado em análise espectral e testes de coerência de sinais de séries históricas de dados geomagnéticos (valores de variação de campo total $(F)$, obtidos no Observatório Magnético de Vassouras (VSS), estado do Rio de Janeiro), e de atividade solar (série de dados de número de manchas solares $\left(R_{Z}\right)$ ), cobrindo intervalos de 89 anos e de 260 anos, respectivamente, com 0 intuito de se observar possíveis padrões correlacionáveis entre feições climáticas e geomagnéticas na região de VSS, possivelmente sob influência da Anomalia Magnética do Atlântico Sul (AMAS). Os resultados obtidos indicam uma ampla faixa de quase-periodicidades, em escala anuais a decadais, indicativas da relação entre a atividade geomagnética e solar sob diferentes processos físicos, e possivelmente associados a mecanismos de oscilação climática de larga escala.
\end{abstract}

\section{Introdução}

Um dos mais debatidos problemas científicos da atualidade se baseia nas evidências de um progressivo aquecimento global, verificado desde o início da Revolução Industrial (Kerton, 2009), que poderia, seguindo a atual tendência, promover um profundo impacto sobre todas as regiões do planeta, como um todo, afetando ecossistemas e a interação entre sociedades em escala global. Neste ínterim, importantes esforços em diferentes linhas de pesquisa vêm sendo conduzidos nas últimas décadas com o intuito de se compreender os processos naturais que poderiam resultar em grandes alterações climáticas - o que é crucial para o importante debate a respeito dos limites da influência antrópica sobre possíveis limiares do sistema climático.

Devido à sua característica não-linear - os processos climáticos são freqüentemente episódicos e abruptos em vez de lentos e graduais, e equilíbrios climáticos múltiplos são sugeridos como norma (Rial et al., 2004) - muitas das hipóteses acerca do clima na Terra ainda carecem de maior entendimento sobre os diferentes tipos de nãolinearidades e como estes fatores se manifestariam sob diferentes condições. Dentre tais problemas em aberto, provavelmente 0 de maior importância se refere à compreensão do sistema climático como sendo dirigido por mecanismos de forçagem por 'feedbacks' internos ou por uma combinação de ambos.

Como exemplo de mecanismos de forçagem, os processos referentes aos ciclos orbitais terrestres são universalmente aceitos e adequadamente descritos pela teoria dos ciclos de Milankovitch, que prediz variações de longo período sobre a quantidade de radiação solar incidente sobre a atmosfera por variações dos parâmetros orbitais. Especificamente com respeito aos possíveis indutores de aumento na temperatura superficial global, são também bastante discutidos a a variabilidade solar (e.g. Hoyt \& Schatten, 1997; Versteegh, 2005), mudanças na concentração de gases de efeito estufa (por ação natural ou antropogênica) e a variabilidade interna do sistema acoplado oceânicoatmosférico, de características não-lineares (e.g. Ganopolski \& Rahmstorf, 2001). Entretanto, é também de consenso na literatura que uma série de questões a respeito dos processos de indução ainda demandam respostas - como exemplo, quais seriam as causas de mudanças climáticas drásticas de curto período, e quais os fatores que geraram curtos episódios glaciais? Em outras palavras, seriam suficientes as flutuações na insolação sazonal, relativamente pequenas, para a explicação da evolução do desenvolvimento das capas de gelo, em escalas de tempo inferiores às sugeridas pela teoria orbital?

Nos últimos anos, o possível papel da atividade geomagnética sobre eventos de mudança climática vem sendo cada vez mais considerado como tópico de importância através de diversos estudos, embora possíveis correlações ainda sejam tópicos de grande controvérsia (Gallet et al., 2005; Fluteau et al., 2006; Pazur \& Winklhofer, 2008). Relações significativas durante os últimos 60 anos foram encontradas entre este tipo de mecanismo e a temperatura atmosférica superficial (e.g. Cliver et al., 1998; Bucha \& Bucha, 1997; Ponyavin, 2004; Le Möuel et al., 2005; Valev, 2006), distribuição de correntes atmosféricas (Bochní ek \& Hejda, 2006) e circulação troposférica, caracterizada pelo índice NAO (sigla em inglês para "Oscilação do Atlântico Norte"; Lukianova \& Alekseev, 2004; Bochní ek \& Hejda, 2006), possíveis influências por modulação 
geomagnética sobre a produção de radionuclídeos cosmogênicos - por sua vez, indicativos para a variabilidade da atividade solar (e.g. Muscheler et al., 2007). Sugestões sobre conexões entre o campo magnético da Terra e o clima também foram propostas por Courtillot et al. (2007) e discutidos criticamente por Bard e Delaygue (2008). Entretanto, como discutido recentemente por Paluš \& Novotná (2009), muitas das relações de dependência observadas são restritas a correlações baseadas em séries de dados relativamente pequenas, fazendo com que a significância estatística das correlações observadas seja muito vulnerável à pequenas mudanças no número analisado de amostras, bem como pela escolha dos parâmetros de préprocessamento (e.g. filtragem ou suavização).

Assim, ainda não se encontra disponível uma explicação de ampla aceitação que explique as respostas troposféricas aos efeitos da atividade geomagnética. De maneira a compreender-se, modelar e predizer tais processos não-lineares, faz-se necessária a identificação dos mecanismos dinâmicos subjacentes aos fenômenos refletidos pelos dados experimentais (Paluš \& Novotná, 2007). Um importante avanço neste sentido pode ser dado por investigações que visem à detecção de padrões, processos oscilatórios e/ou sinais potencialmente determinísticos pela correlação de dados meteorológicos e geomagnéticos.

Neste ínterim, estudos de larga escala espacial e que possam compreender um período relativamente longo de tempo em cobertura de dados - com o intuito de se verificar variabilidades de curto período do campo geomagnético e suas possíveis interações com processos climáticos - apresentam uma enorme potencialidade para importantes avanços com respeito aos debates que vem sendo colocados pela comunidade científica.

No presente trabalho, apresentaremos resultados preliminares de um estudo baseado em séries históricas de dados geomagnéticos (valores de campo total $(F)$, medidos no Observatório Magnético de Vassouras (VSS), estado do Rio de Janeiro), e de atividade solar (série de dados de número de manchas solares $\left(R_{Z}\right)$ ), cobrindo um intervalo de 89 anos e de 260 anos, respectivamente, com 0 intuito de se observar possíveis padrões correlacionáveis entre feições climáticas e geomagnéticas na região de VSS, possivelmente sob influência da Anomalia Magnética do Atlântico Sul (AMAS).

\section{Metodologia}

Aquisição de dados

Para a aquisição da maioria dos dados geomagnéticos empregados neste trabalho, procedemos com a digitalização de compilação de dados provenientes de observações geomagnéticas realizadas pelo Observatório Magnético de Vassouras, e que são periodicamente publicados pelo Observatório Nacional (ON-MCT). Foi definida para este trabalho uma série de dados compreendendo os anos de 1915 e 2004, correspondente ao período de maior cobertura de dados e com o menor número de interrupções. Eventuais lacunas nas séries de dados foram completadas com o acesso a registros já digitalizados pelo corpo técnico do ON-MCT.

Para este trabalho, foram adotadas as seguintes componentes geomagnéticas: campo total (F), componente horizontal $(\mathrm{H})$ e componente vertical $(\mathrm{Z})$ do campo geomagnético. Os dados direcionais ( $\mathrm{He} \mathrm{Z}$ ) foram empregados para o cálculo da componente de campo total para o período em que a aquisição direta de $\mathrm{F}$ não era realizada. Além disso, as séries de dados de $F$ empregadas neste estudo (Fig. 1) - e compreendidas para o intervalo entre 1915 e 2004 foram preparadas através de quatro resoluções temporais distintas: mensal, trimestral, semestral e anual, com o objetivo de se observar possíveis padrões harmônicos, que possam ser relacionáveis a determinados processos climáticos e que operem em distintos intervalos de tempo.

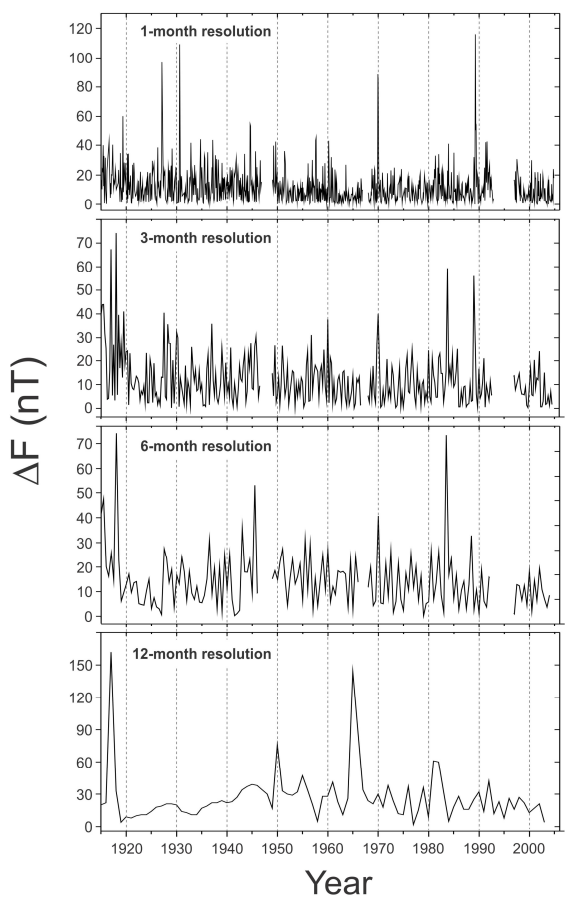

Figura 1: Séries de dados de variação de campo total

$(\Delta \mathrm{F})$ nas quatro resoluções adotadas.

Os dados referentes ao número de manchas solares (Fig. 2) foram obtidos a partir do banco de dados disponibilizado online pelo National Geophysics Data Center (NGDC), EUA, através do sítio (http://www.ngdc.noaa.gov/nndc/struts/results? $\mathrm{t}=102827 \&$ $\underline{\mathrm{s}=5 \& \mathrm{~d}=8,430,9}$ ). A série de dados obtida para este trabalho corresponde, como discutido anteriormente, aoperíodo entre 1749 a 2009. O ciclo de manchas solares - grandes perturbações eletromagnéticas na superfície solar (Niroma, 2009), cujo período é um indicador da atividade erupcional do Sol (Landscheidt, 2003) foi escolhida para os procedimentos de análise espectral, que apresentaremos na próxima seção. 


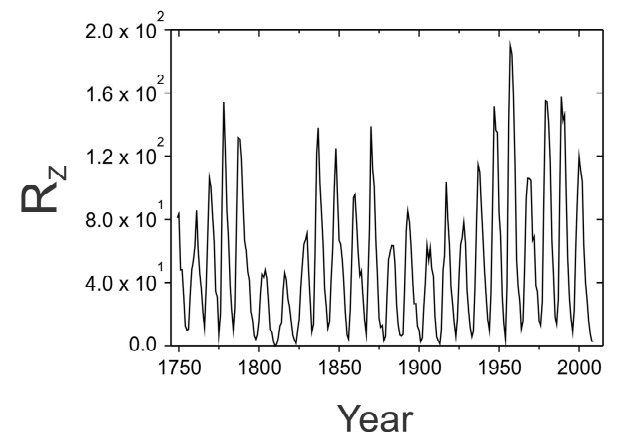

Figura 2:

Médias

anuais do

número de

manchas

solares $\left(R_{\mathrm{Z}}\right)$

(National

Geophysics

Data Center

(NGDC)

EUA).

\section{Análise espectral}

Devido às lacunas presentes nas séries de dados geomagnéticos, o processo de análise espectral para as séries temporais foi realizado com o emprego do software SPECTRUM, (Schulz \& Stattegger, 1997), que se baseia na Transformada de Fourier de Lomb-Scargle, (comumente empregada em análise de sinais para séries de dados desigualmente espaçados), em combinação com o método WOSA ("Welch-Overlapped-SegmentAveraging"; Welch, 1967; Tröbs \& Heinzel, 2006), que por sua vez permite a supressão de picos aleatórios ou 'outliers' através da divisão da série temporal, dentro dos segmentos superpostos e tomando a média do espectro calculado.

Após a eliminação de tendências, cada série de dados foi dividida em segmentos (2 ou 3 segmentos), dependendo da quantidade de dados disponíveis - e superpostos em $50 \%$. Em seguida, cada segmento foi multiplicado por uma função janela do tipo Welch I (Harris, 1978), para a redução dos efeitos relacionados à dispersão espectral, sendo posteriormente processados por transformada de Fourier. Os periodogramas assim obtidos permitem 0 cálculo do periodograma médio, que é associado à sua largura de banda de $6 \mathrm{~dB}$ e nível de significância $\alpha$ (definido neste trabalho em 0,05). Além disso, foi calculado o espectro de coerência para as séries de dados de $\mathrm{F}$ e $\mathrm{R}_{\mathrm{Z}}$ de maneira a verificar possíveis padrões harmônicos presentes em ambas as séries, e que sejam possívelmente relacionados.

\section{Resultados e Conclusões}

A seguir, apresenta-se a figura 3, que dispõe os autoespectros provenientes da análise espectral para séries desigualmente espaçadas no domínio do tempo, constituídas sob quatro distintas resoluções temporais (conforme apresentadas na figura 2), e associadas a níveis de confiança de 95\%.

A série de dados de $\Delta \mathrm{F}$ mensais e trimestrais revelam diferentes modos oscilatórios através de vários picos espectrais acima do nível de confiança de 95\% (Fig. 3-A e 3-B, respectivamente), o que poderia ser explicado pelo pequeno valor de largura de banda de $6 \mathrm{~dB}$ devido à alta resolução obtida. É possível distinguir claramente, para estes dois espectros, quatro faixas de valores: (i) 0,21-yr - 0,29-yr; (ii) 0,33-yr - 0,44-yr; (iii) 0,5-yr - 0,65-yr; (iv) 0,80-yr - 1,12-yr. A faixa de picos espectrais (i), de menor potência espectral que as demais, é sugestiva do primeiro harmônico da variação semi-anual de atividade geomagnética, de período aproximadamente igual a 6 meses e que, por sua vez, é claramente observada pela faixa de picos (iii) - valores correspondentes a 0,5-yr (Fig. 3-A), 0,61-yr e 0,65-yr (Fig. 3-B).

Recentemente, Prestes et al. (2006) sugeriram, através de análise espectral de séries de índice de atividade antipodal geomagnética, fortes evidências para o registro da variação semi-anual de atividade geomagnética pela presença de um pico espectral de 0,5 anos, e que é caracterizada por uma maior geoefetividade em períodos equinociais que em períodos de solstício.

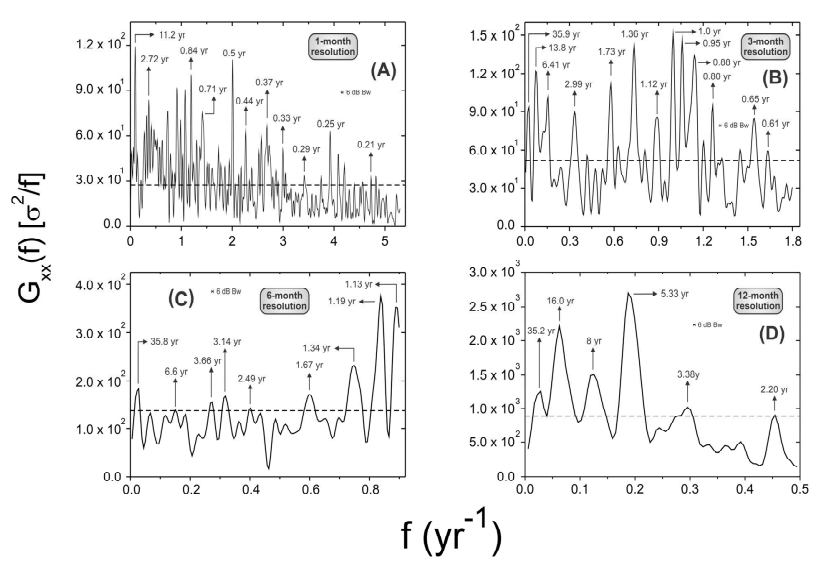

Figura 3: Espectros de potência das séries temporais de variação de campo total $(\Delta \mathrm{F})$, em resolução mensal $(A)$, trimestral $(B)$, semestral (C) e anual (D), associados ao nível de confiança de $95 \%$ (linha tracejada).

Pela figura $3-B$, ainda é possível notar-se três picos espectrais (1,12-yr, 1,36-yr e 1,73-yr), apresentando o segundo pico maior potência espectral que os demais. Alguns autores (e.g. Paularena et al., 1995; Krivova \& Solanki, 2002) discutem uma periodicidade de 1,3 anos na taxa de rotação solar próxima à base da zona de convecção solar, e que é discutida como compatível com a intensidade do campo magnético interplanetário (IMF; Kudela et al., 2002). Segundo Mursula \& Zieger (2000), a variação de 1,3 anos corresponde a uma quaseperiodicidade que ocorre durante ciclos solares pares, enquanto que, para ciclos ímpares, verificam-se periodicidades mais longas com períodos na faixa entre 1,5 a 1,7 anos, e que seriam consideradas devido à evolução de buracos coronais - tais diferenças em período, observáveis em registros geomagnéticos, revelariam diferenças na evolução destas feições solares durante ciclos solares pares e ímpares.

Não é direta a explicação para a ocorrência dos picos da faixa (ii) e (iv) - entretanto, pode-se sugerir para a ocorrência do primeiro grupo de modos oscilatórios como relacionada à modulação da atividade geomagnética pela atividade solar, devido à sazonalidade determinada pelas estações do ano. Entretanto, alguns estudos (e.g. Krivova $\&$ Solanki, 2002) discutem que a variabilidade solar de 
154-158 dias, conhecida como período de Rieger de explosões solares - portanto compatível com o pico espectral de 0,44-yr da faixa (ii) - corresponderia ao terceiro harmônico da quase-periodicidade solar de 1,3 anos.

Os picos espectrais na faixa entre 2,0-yr - 3,66-yr, que são observados em todos os espectros de potência, são bastante similares a padrões harmônicos que vêm sendo discutidos na literatura como oscilações quase-bienais (QBO) e quase-trienais (QTO) em estudos sobre séries temporais de índices geomagnéticos, possivelmente correlacionados com processos físicos solares relacionados à periodicidade na emissão de ventos solares (e.g. Kane, 1997; Prestes et al., 2006; Paluš \& Novotná, 2006), e que até mesmo têm sido sugeridos como possivelmente relacionados à variabilidade em índices pluviométricos (Kane, 1998).

Os picos espectrais de 6,41-yr (Fig. 3-B), 6,60-yr (Fig. 3C) e 5,33-yr (Fig. 3-D) poderiam ser interpretados como o primeiro harmônico do ciclo solar de 11 anos. Este ciclo pode se apresentar através de picos espectrais em uma faixa de 8 a 13,5 anos (e.g. Prestes et al., 2006, Niroma, 2009) - o que explicaria a variabilidade de valores referentes a este harmônico, bem como os picos espectrais de 11,2-yr e 13,8-yr (Figs. 3-A e 3-B, respectivamente), ilustrativos da variabilidade para este ciclo solar, e que também pode ser claramente observado pela espectro de potência para a série de $R_{Z}$ (Fig. 4). Entretanto, uma periodicidade de $~ 5.3$ anos vem sendo sugerida por alguns autores (e.g. Dmitriev et al., 2000) como sendo referente a um padrão harmônico relativo à densidade do vento solar, e que é conhecida

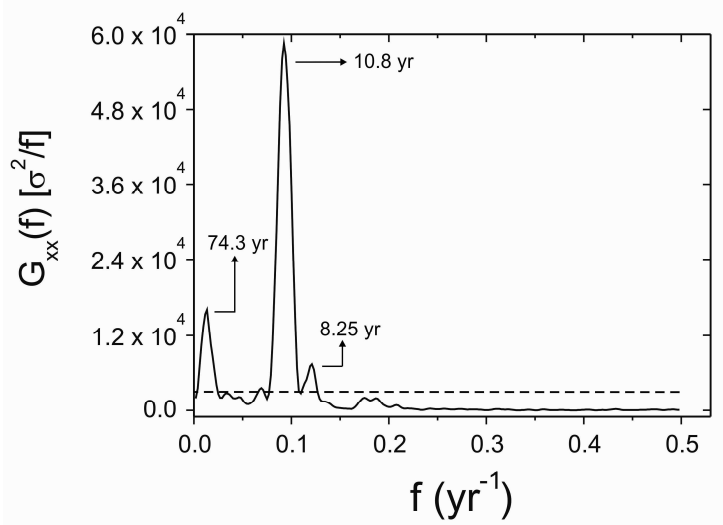

Figura 4: Espectro de potência da série temporal do número de manchas solares $\left(R_{z}\right)$, associado ao nível de confiança de $95 \%$ (linha tracejada)

como um dos fatores mais importantes sobre o controle da atividade geomagnética (Stamper et al., 1999). Ainda é importante salientar que, na figura 4, também é possível identificar-se a presença de um pico espectral igual a 74,3-yr, e que pode ser associada ao ciclo heliomagnético de Wolf-Gleissberg - quase-periodicidade na faixa de 80 a 120 anos (e.g. Braun et al., 2005; Yousef, 2006) sugerida como resultado da modulação de amplitude do ciclo de 11 anos (Sonnett \& Finney, 1990).
Não é possível uma inferência direta para a periodicidade observada, de cerca de 35 anos, bastante persistente como sinal harmônico nos espectros de potência para as séries de $\Delta \mathrm{F}$ (Figs. 3-B a 3-D). Este período foi discutido recentemente por Prestes et al. (2006) para espectros de amplitude de índices aa e Ap, restritos a intervalos de alta atividade solar, embora os autores não tenham sugerido nenhuma relação causal direta. Curiosamente, a variabilidade temporal da Oscilação Decadal do Pacífico - padrão persistente da variabilidade do Pacífico Norte (Tanimoto et al., 1993; Zhang et al., 1997) - é vinculada a um ciclo de 35,8 anos, e que teria relação, segundo Landscheidt (1998; 2000; 2003), com perturbações no movimento solar em relação ao centro de massa do Sistema Solar. Ainda segundo este autor, a taxa de variação do momento angular orbital solar gera um período de torque de aproximadamente 16 anos claramente compatível com o pico espectral de mesmo valor, observado na fig. 3-D.

De maneira a investigar possíveis padrões harmônicos comuns às séries de dados temporais de $\Delta F$ e $R_{z}$, efetuamos um teste de coerência (Fig. 5) associado a um nível de alarme falso de $90 \%$, segundo algoritmo discutido por Schulz e Stattegger (1997), após a restrição da série de $R_{z}$ para o mesmo intervalo compreendido pela série de dados geomagnéticos (1915 a 2004).

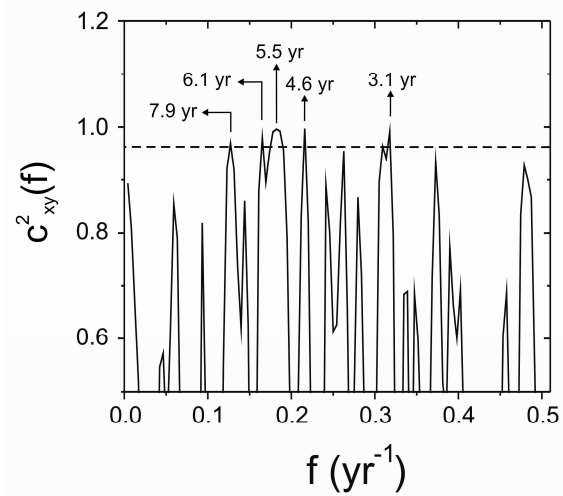

Figura 5: Teste de coerência associado a um nível de alarme falso de $90 \%$ (linha tracejada) para as séries de variação de campo total $(\Delta \mathrm{F})$ e de número de manchas solares $\quad\left(\mathrm{R}_{\mathrm{z}}\right)$ para o período entre 1915 e 2004.

Os resultados obtidos através do teste de coerência se baseiam claramente em algumas das quaseperiodicidades em escala sub-decadal aqui discutidas, indicando a QTO (3,1-yr), a periodicidade de 5,5-yr, e o primeiro harmônico do ciclo de 11 anos.

Com respeito ao sinal verificado para 7,9 anos, determinados modos oscilatórios, com periodicidades de aproximadamente $7-8$ anos foram observados em séries temporais de números de manchas solares, atividade geomagnética, índices NAO e temperatura do ar próxima à superfície para várias localidades em média latitude na Europa (Paluš \& Novotná, 2009). Além disso, alguns autores (e.g. Chu, 1984; Kane, 1998) observaram um padrão similar ao sinal de 4,6 anos (em torno de 4,5 5 anos) como proeminente em todos os parâmetros relacionados à precipitação no nordeste brasileiro, e um sinal persistente de 5,5 anos com respeito à temperatura superficial do mar (SST) no Atlântico Sul (Kane, 1998) 
que é, por sua vez, fortemente relacionada ao ENSO modo global primário de variabilidade climática que ocorre em uma escala temporal quase-periódica de $2-7$ anos (Mann et al., 2005; Vieira \& Silva, 2006).

Tais periodicidades, em torno de 3,0 - 5,0 anos ocorrem como variabilidades associadas ao vento solar e fase de atividade de manchas solares, e também observáveis na polaridade do IMF - que tem origem em processos físicos solares e que são bastante compatíveis com os padrões harmônicos em torno de 3,5 anos relacionados ao ENSO (Kane, 1997).

Embora, no estágio atual deste projeto de pesquisa, não seja possível a proposição de um mecanismo plausível que sugira uma conexão direta entre forçagem climática e geomagnética, os resultados encontrados são bastante sugestivos de uma interdependência entre a variabilidade do campo total com relação a determinadas feições de atividade solar em escalas sub- a decadais, e que vêm sendo discutidas na literatura como fortemente relacionadas a mecanismos de oscilação climática de larga escala. Tais resultados claramente apontam para a necessidade de maiores investigações, de maneira a verificar possíveis similaridades discutidas por este estudo em diferentes áreas do território nacional, relacionáveis a distintas feições geomagnéticas.

\section{Agradecimentos}

Os autores agradecem ao corpo técnico do Observatório Nacional - em especial ao Sr. Ronaldo de Carvalho pela disponibilização dos dados geomagnéticos do Observatório Magnético de Vassouras. FCLS e SLF agradecem ao CNPq pela bolsa de mestrado e produtividade. DRF tem bolsa de Pesquisador Visitante CAPES-FAPERJ.

\section{Referências}

Bard, E., Delaygue, G., Comment on "Are there connections between the Earth's magnetic field and climate?" by V. Courtillot, Y. Gallet, J.-L. Le Mouel, F. Fluteau, A. Genevey EPSL 253, 328, 2007. Earth Planet. Sci. Lett. 265, 302-307 (2008).

Bochní ek, J., Hejda, P., Connections between the distribution of prevailing winds in the winter Northern Hemisphere, solar/geomagnetic activity and the QBO phase, Stud. Geophys. Geod. 50 (2), 299-318 (2006).

Braun, H., Christl, M., Rahmstorf, S., Ganopolski, A., Mangini, A., Kubatzki, C., Roth, K., Kromer, B., Possible solar origin of the 1,470-year glacial climate cycle demonstrated in a coupled model, Nature 438, 208-211 (2005).

Bucha, V., Bucha, V., Geomagnetic forcing of changes in climate and in the atmospheric circulation. J. Atmos. Sol. Terr. Phys., 60 (2), 145-169 (1997).
Bucha, V., Bucha, V., Geomagnetic forcing and climatic variations in Europe, North America and in the Pacific Ocean, Quat. Int. 91 (1), 5-15 (2002).

Chu, P.S., Time and space variability of rainfall and surface circulation in the northeast Brazil - tropical Atlantic sector, J. Meteor. Soc. Japan 62, 363-370 (1984).

Cliver, E.W., Boriakoff, V., Feyman, J., Solar variability and climate change: geomagnetic aa index and global surface temperature. Geophys. Res.Lett. 25 (2), 10351038 (1998).

Courtillot, V., Gallet, Y., Le Mouël, J.-L., Fluteau, F., Genevey, A., Are there connections between the Earth's magnetic field and climate? Earth Planet. Sci. Lett. 253, 328-339 (2007).

Dmitriev, A.V., Suvorova, A.V., Veselovsky, I.S., Solar wind and interplanetary magnetic field parameters at the Earth's orbit during three solar cycles, Phys. Chem. Earth (C) 25, 125-128 (2000).

Dobrica, V., Demetrescu, C., Boroneant, C., Maris, G., Solar and Geomagnetic activity effects on climate at regional and global scales: Case study - Romania, J. Atmos. Sol. Terr. Phys. 71 (17-18), 1727-1735 (2009).

Ganopolski, A., and Rahmstorf, S., Rapid changes of glacial climate oscillations simulated in a coupled climate model, Nature, 409, 153-158, 2001.

Harris, F.J., On the use of windows for harmonic analysis with the discrete Fourier Transform, Proc. IEEE 66 (1), 51-83 (1978).

Hoyt, D.V., Schatten, K.H., 1997. The Role of the Sun in Climate Change. Oxford University Press, New York, 279 pp.

Kane, R.P., Quasi-biennial and quasi-triennial oscillations in geomagnetic activity indices, Ann. Geophysicae 15, 1581-1594 (1997).

Kane, R.P., Quasi-biennial and quasi-triennial oscillations in the rainfall of Northeast Brazil, Rev. Bras. Geofis. 16 (1), 37-52 (1998).

Kerton, Adrian K., Climate Change and the Earth's Magnetic Poles, a Possible Connection, Energy \& Environment, Volume 20, Numbers 1-2, January 2009, pp. 75-83(9).

Krivova, N.A., Solanki, S.K., The 1.3-year and 156-day periodicities in sunspot data: Wavelet analysis suggests a commom origin, Astron. Astrophys. 394, 701-706 (2002).

Kudela, K., Rybák, J., Antalová, A., Storini, M., Time Evolution of Low-Frequency Periodicities in Cosmic Ray Intensity, Sol. Phys. 205, 165-1475 (2002).

Landscheidt, T., Solar activity - A dominant factor in climate dynamics. http://www.johndaly.com/solar/solar.htm. (1998):

Landscheidt, T., River Po discharges and cycles of solar activity. Hydrol. Sci. J. 45, 491-493 (2000). 
Landscheidt, T., New Little Ice Age instead of Global Warming?, Energy Environ. 14 (2-3), 327-350 (2003).

Le Möuel, J.L., Kossobokov, V., Courtillot, V., On long term variations of simple geomagnetic indices and slow changes in magnetospheric currents: the emergence of anthropogenic global warming after 1990? Earth Planet. Sci. Lett. 232(3-4), 273-286 (2005).

Lukianova, R., Alekseev, G., Long-term correlation between the NAO and solar activity. Sol. Phys. 224, 445454 (2004).

Mann, M.E., Cane, M.A., Zebiak, S.E., Clement, A., Volcanic and Solar Forcing of the Tropical Pacific over the Past 1000 Years, J. Climate 18 (3) 447-456 (2005).

Mursula, K., Zieger, B., The 1.3-year variation in solar wind speed and geomagnetic activity, Adv. Space Res. 25 (9), 1939-1942 (2000).

Niroma, T., Understanding solar behaviour and its influence on climate, Energy Environ. 20 (1-2), 145-159 (2009).

Ponyavin, D.I., Solar cycle signal in geomagnetic and climate, Sol. Phys. 224 (1), 465-471 (2004).

Prestes, A., Rigozo, N.R., Echer, E., Vieira, L.E.A., Spectral analysis of sunspot number and geomagnetic índices (1868-2001), J. Atmos. Sol. Terr. Phys. 68, 182190 (2006).

Rial, J., Abrupt climate change: chaos and order at orbital and millennial scales. Global. Planet. Change, 41, 95-109 (2004).

Paluš, M., Novotná, D., Quasi-biennial oscillations extracted from the monthly NAO index and temperature records are phase-synchronized, Nonlinear Processes Geophys. 13, 287-296 (2006).

Paluš, M., Novotná, D., Common oscillatory modes in geomagnetic activity, NAO index and surface air temperature records, J. Atmos. Sol. Terr. Phys., 69, 2405-2415 (2007).

Paluš, M., Novotná, D., Phase-coherent oscillatory modes in solar and geomagnetic activity and climate variability, $\mathrm{J}$. Atmos. Sol. Terr. Phys., 71, 923-930 (2009).

Paularena, K. I., Szabo, A., Richardson, J.D., Coincident 1.3-year periodicities in the ap geomagnetic index and the solar wind, Geophys. Res. Lett. 22 (21), 3001-3004 (1995).

Schulz, M., Stattegger, K., Spectrum: Spectral Analysis of Unevenly Spaced Paleoclimatic Time Series, Computers \& Geosciences, Volume 23, Issue 9, Pages 929-945, (1997).

Sonnett, C.P., Finney, S.A., The spectrum of radiocarbon, Philos. Trans. R. Soc. London, Ser. A (30), 413-426 (1990).

Stamper, R., Lockwood, M., Wild, M.N., Clark, T.D.G., Solar causes of the long-term increase in geomagnetic activity, J. Geophys. Res. 104, 28325-28342 (1999).
Tanimoto, Y.N., Iwasaka, N., Hanawa, K., Toba, Y., Characteristic variations of sea surface temperature with multiple time scales in the North Pacific. J. Climate 6, 1153-1160 (1993).

Tröbs, M., Heinzel, G., Improved spectrum estimation from digitized time series on a logarithmic frequency axis, Measurement 39, 120-129 (2006).

Valev, D., Statistical relationships between the surface air temperature anomalies and the solar and geomagnetic activity indices. Phys. Chem. Earth 31 (1-3), 109-112 (2006).

Versteegh, G.J.M., Solar forcing of climate. 2: Evidence from the past, Space Sci. Rev. 120, 243-286 (2005).

Welch, P.D., The use of fast Fourier transform for the estimation of power spectra: A method based on time averaging over short, modified periodograms, IEEE Trans. Audio Electroacoust. 15 (2), 70-73 (1967).

Yousef, S.M., 80-120 yr Long-term solar induced effects on the earth, past and predictions, Phys. Chem. Earth 31, 113-122 (2006).

Zhang,Y., Wallace, J. M., Battisti, D. S., ENSO-like interdecadal variability: 1900-1993. J. Climate 10, 10041020 (1997). 